\title{
Possible factors influencing postoperative temporary neurologic deterioration following standard superficial temporal artery-middle cerebral artery (STA-MCA) bypass surgery: diameter of STA and MCA (M4)
}

\author{
Jie Bai, MD, PhD ${ }^{1}$; Yuan-Li Zhao, MD, $\mathrm{PhD}^{2, *}$; Ji-Zong Zhao, MD, $\mathrm{PhD}^{2}$; Rong Wang, MD, $\mathrm{PhD}^{2}$;Dong \\ Zhang, MD,PhD ${ }^{2}$;Song Lin , MD, PhD $^{2}$; Chang-Wu Dou, MD,PhD ${ }^{1}$ \\ ${ }^{1}$ Department of Neurosurgery, Affiliated Hospital of Inner Mongolia Medical College, Inner Mongolia Medical College, \\ 010050 , Inner Mongolia, China
}

${ }^{2}$ Department of Neurosurgery, Beijing Tiantan Hospital, Capital Medical University, 100050, Beijing, China

\begin{abstract}
Objectives: Transient postoperative hyperperfusion may be the cause of the temporary neurologic deterioration (TND) frequently observed following superficial temporal artery-middle cerebral artery (STA-MCA) bypass surgery. The purpose of this study is to investigate the possible factors influencing postoperative temporary neurologic deterioration.
\end{abstract}

Methods: The STA-MCA bypass surgery was performed in 13 patients with moyamoya disease and 9 patients with cerebrovascular occlusive disease. The diameter of the STA and MCA for anastomosis was measured and analyzed. Computed tomographic perfusion (CTP) was performed prior to the operation and on the third postoperative day to assess improvements in regional cerebral perfusion.

Results: After surgery, five patients (22.7 \%) with improvement of perfusion suffered TND such as dysphasia, motor deficit and sensory disturbance after surgery and $11(50 \%)$ patients with improvement of perfusion did not show temporary neurologic deterioration after surgery. There were six patients $(27.3 \%)$ without improvement of perfusion after surgery. The D-STA was $1.48 \pm 0.92 \mathrm{~mm}$ in patients with TND, which was larger than the D-STA of $1.10 \pm 0.14 \mathrm{~mm}$ in patients with improvement of perfusion and $1.08 \pm 0.26 \mathrm{~mm}$ in patients without improvement of perfusion $(P<0.05)$; The D-MCA after surgery was also wider in patients with TND (DMCA: $1.20 \pm 0.19 \mathrm{~mm}$ ) compared to both patients with hyperperfusion (D-MCA: $0.93 \pm 0.15 \mathrm{~mm}, p=0.028$ ) and patients without hyperperfusion (D-MCA: $0.98 \pm 0.12 \mathrm{~mm}, p=0.012$ ). The TOT was $34.40 \pm 7.70$ minutes in patients with TND, $32.83 \pm 3.43$ minutes in patients with improvement in perfusion and $33.45 \pm 4.48$ minutes in patients without improvement of perfusion; there was no significant difference between the three groups $(P>0.05)$.

Conclusions: The D-STA and D-MCA may influence the development of TND after STA-MCA bypass, but the TOT of the recipient arteries was not related to temporary neurologic deterioration.

Key words: Moyamoya disease; Temporary neurologic deterioration; Transient hyperperfusion; STA-MCA bypass; Diameter of STA

\section{Introduction}

Superficial temporal artery-middle cerebral artery (STA-MCA) bypass surgery is the main surgical treatment for chronic occlusive cerebrovascular disease. It can prevent cerebral ischemic attacks by improving regional cerebral blood flow (CBF). However, the change in CBF can affect neurologic status during the acute stage after the direct bypass. Increasing evidence suggests that direct revascularization surgery for moyamoya disease can result in temporary neurologic deterioration (TND) owing to focal cerebral hyperperfusion, with an incidence of $4-20 \%$. TND is characterized by headache, dysphasia, seizures, motor deficits of the limbs and sensory disturbance in the contralateral upper limb and/or face (SensD). These symptoms can be relieved by intensive blood pressure control. Preoperative cerebrovascular reserve capacity, severity of ischemia during surgery, and anatomical vascular structures around the site of the anastomosis may affect postoperative cerebral hyperperfusion and cause TND. The diameter of the STA (D-STA) and MCA-M4 (D-MCA) and temporal occlusion times (TOT) of the recipient artery mainly determine the vascular structures around the site of the anastomosis during surgery and are possible factors influencing the development of postoperative TND in STA-MCA bypass surgery.

To address this issue, we retrospectively investigated D-STA, D-MCA and TOT in 22 consecutive patients with chronic ischemic cerebrovascular disease who underwent STA-MCA bypass. The regional cerebral

*Correspondence should be sent to:

Yuan-Li Zhao, Department of Neurosurgery, Beijing Tiantan Hospital, Capital Medical University,100050, Beijing, China. E-mail: dr.zhaoyuanli@gmail.com 
perfusion was examined by computed tomographic perfusion (CTP) both pre-operatively and on the third post-operative day.

\section{Materials and Methods}

\subsection{Population and indications for STA-MCA bypass}

The study included 13 patients with moyamoya disease (MMD) and 9 patients with cerebrovascular occlusive disease (CVO) who were diagnosed in our hospital. The male-to-female patient ratio was $6: 16$, and the mean age was 38.1 years (range, 14-56 years) (Table 1). Bilateral carotid and vertebral angiography was performed on all patients. All 22 patients underwent unilateral standard STA-MCA bypass surgery. The indications for STA-MCA bypass and surgical side were determined by clinical symptoms, preoperative digital subtraction angiography (DSA) and CTP. The patients who conformed simultaneously to the following three items were considered for STA-MCA bypass: 1. Patients with clinical symptoms such as transient ischemic attack (TIA), infarction and hemorrhage; 2. Unilateral or bilateral intracranial vascular occlusion confirmed by preoperative DSA; 3 . Regional cerebral hypotension confirmed by CTP and consistent with clinical symptoms. The choice of surgical side was made according to the following criteria: 1 . Unilateral intracranial vascular occlusion, perform the bypass on the lesioned side; 2.Bilateral intracranial vascular occlusion, perform the bypass on the side leading to symptoms; 3. Bilateral regional cerebral hypotension, perform the bypass on the side leading to symptoms; 4. Symptoms on bilateral sides, perform the bypass on the side leading to more serious symptoms and consider the other side for a delayed operation.

\subsection{Surgical procedure}

The surgical procedure was standard STA-MTA bypass. We generally separated the frontal branch and parietal branch of the STA, chose the best one (longer and with a good pulse) for anastomosis, and electrocoagulated the other branch. The STA was anastomosed intermittently to the MCA (M4) in and end-to-side manner using 10-0 nylon suture. The patency of the anastomotic site was confirmed by intraoperative indocyanine green angiography (ICGA). The temporal occlusion times of the recipient artery were recorded. The D-STA and D-MCA were measured as follows: When the STA and the branch of MCA were completely exposed and occluded, we divested the vascular adventitia, heparin lavaged the vessels to remove thromboses and continuously douched vessels with papaverine to releasing the spasm, then measured the D-STA and D-MCA with microcalipers.

\subsection{Assessment of regional cerebral perfusion}

To assess improvement after surgery, the CTP was performed by generating maps of cerebral blood flow (CBF) and cerebral blood volume (CBV) preoperatively and on the third post-operative day. We chose a comparison to the contralateral side of the brain to evaluate changes in CTP. Through comparison of the CBF and CBV on the surgical side and the contralateral side preoperatively and postoperation, we confirmed the change in regional cerebral perfusion.

\subsection{Statistical analysis}

Data were expressed as mean \pm SD and statistically analyzed using a nonparametric Wilcoxon signed rank test between two groups and one-way ANOVA followed by post hoc Fisher's or Kruskal-Wallis test. All analysis was performed using a software program (SPSS 11.0). $P<0.05$ was considered statistically different.

\section{Results}

3.1. The transient improvement of perfusion and TND after STA-MCA bypass surgery

Unilateral STA-MCA bypass was performed in 22 consecutive patients. Based on comparison of perfusion computed tomography prior to the operation and on the third postoperative day, five patients (22.7 \%) with improvement of perfusion suffered TND such as dysphasia, motor deficit and sensory disturbance after surgery and $11(50 \%)$ patients with improvement of perfusion did not show TND after surgery. There were six patients $(27.3 \%)$ without improvement of perfusion after surgery (Table 1). TND occurred from the first day or second day after surgery, and was sustained for 6 - 7 days in three patients and for 20 days in one patient (Table 1). The anatomical location and the temporal profile of hyperperfusion were completely in accordance with the symptoms of TND in five patients. Symptoms were relieved by treatment of intensive blood pressure control and no patients remained permanently neurologically impaired. 
Table1 clinical features and postoperative perfusion in 22 patients

\begin{tabular}{|c|c|c|c|c|c|c|c|c|c|c|}
\hline $\begin{array}{l}\text { Case } \\
\mathrm{N}\end{array}$ & $\begin{array}{l}\text { Age/s } \\
\text { ex }\end{array}$ & $\begin{array}{l}\text { Diagnos } \\
\mathrm{e}\end{array}$ & $\begin{array}{l}\text { Preoperative } \\
\text { presentation }\end{array}$ & $\begin{array}{l}\text { Operati } \\
\text { ve side }\end{array}$ & $\begin{array}{l}\text { Symptoms of } \\
\text { TND }\end{array}$ & $\begin{array}{l}\text { Period of } \\
\text { deficit }\end{array}$ & $\begin{array}{l}\text { D-STA } \\
(\mathrm{mm})\end{array}$ & $\begin{array}{l}\text { D-MCA } \\
(\mathrm{mm})\end{array}$ & $\begin{array}{l}\text { TOT } \\
\text { (minute) }\end{array}$ & $\begin{array}{l}\text { Hyperperfusion } \\
\text { by CTP }\end{array}$ \\
\hline 2 & $42 / \mathrm{F}$ & MMD & Infarction, TIA & Right & SensD & POD 1-7 & 1.3 & 1.3 & 27 & Yes \\
\hline 4 & $32 / F$ & MMD & TIA & Right & No & No & 1.1 & 1.0 & 30 & Yes \\
\hline 5 & $35 / F$ & MMD & Infarction, TIA & Right & No & No & 0.9 & 1.0 & 40 & Yes \\
\hline 6 & $41 / F$ & MMD & $\mathrm{TIA}$ & Left & No & No & 1.2 & 1.0 & 37 & Yes \\
\hline 8 & $32 / M$ & MMD & $\begin{array}{l}\text { Hemorrhage, } \\
\text { TIA }\end{array}$ & Right & No & No & 0.9 & 1.0 & 35 & No \\
\hline 9 & $33 / F$ & MMD & TIA & Left & $\begin{array}{l}\text { Motor deficit, } \\
\text { SensD }\end{array}$ & POD 2-7 & 1.5 & 1.0 & 40 & Yes \\
\hline 10 & $54 / F$ & MMD & $\begin{array}{l}\text { Hemorrhage, } \\
\text { TIA }\end{array}$ & Right & No & No & 1.1 & 1.1 & 35 & Yes \\
\hline 11 & $14 / F$ & MMD & Infarction, TIA & Right & No & No & 1.4 & 1.0 & 35 & Yes \\
\hline 15 & $54 / F$ & CVO & Infarction, TIA & Right & $\begin{array}{l}\text { Dysphasia, } \\
\text { Motor deficit, } \\
\text { SensD }\end{array}$ & $\begin{array}{l}\text { POD 1- } \\
20\end{array}$ & 1.8 & 1.4 & 30 & Yes \\
\hline 16 & $47 / F$ & CVO & Infarction, TIA & Right & No & No & 1.0 & 1.0 & 30 & Yes \\
\hline 17 & $49 / \mathrm{M}$ & CVO & Infarction & Right & No & No & 0.8 & 0.8 & 35 & No \\
\hline 18 & $41 / F$ & CVO & Infarction, TIA & Right & No & No & 1.0 & 1.0 & 28 & No \\
\hline 19 & $42 / \mathrm{F}$ & CVO & TIA & Right & $\begin{array}{l}\text { Dysphasia, } \\
\text { SensD }\end{array}$ & POD 2-7 & 1.4 & 1.3 & 30 & Yes \\
\hline 20 & $39 / F$ & CVO & Infarction & Left & No & No & 1.5. & 0.7 & 37 & No \\
\hline 21 & $34 / F$ & CVO & $\begin{array}{l}\text { Hemorrhage, } \\
\text { TIA }\end{array}$ & Right & No & No & 1.2 & 1.2 & 35 & Yes \\
\hline 22 & $56 / \mathrm{M}$ & CVO & Infarction & Right & No & No & 1.0 & 1.0 & 28 & Yes \\
\hline
\end{tabular}

3.2. Possible factors influencing on postoperative TND in STA-MCA bypass

The D-STA was $1.48 \pm 0.92 \mathrm{~mm}$ in patients with TND, which was larger than the D-STA of $1.10 \pm 0.14 \mathrm{~mm}$ in patients with improvement of perfusion and $1.08 \pm$ $0.26 \mathrm{~mm}$ in patients without improvement of perfusion $(P<0.05)$; The D-MCA after surgery was also wider in patients with TND (D-MCA: $1.20 \pm 0.19 \mathrm{~mm}$ ) compared to both patients with hyperperfusion (DMCA: $0.93 \pm 0.15 \mathrm{~mm}, p=0.028$ ) and patients without hyperperfusion (D-MCA: $0.98 \pm 0.12 \mathrm{~mm}, p=$ 0.012 )(Table 2). The TOT was $34.40 \pm 7.70$ minutes in patients with TND, $32.83 \pm 3.43$ minutes in patients with improvement in perfusion and $33.45 \pm 4.48$ minutes in patients without improvement of perfusion; there was no significant difference between the three groups $(P>0.05)($ Table 2$)$. There were no statistically significant differences in D-STA, D-MCA and TOT between the patients with and without improvement in perfusion after surgery (Table 3).

Table 2. Possible influence factors for postoperative TND in STA-MCA bypass (mean \pm SD).

\begin{tabular}{lllll}
\hline Postoperative perfusion & Cases $(\mathrm{n})$ & D-STA $(\mathrm{mm})$ & D-MCA $(\mathrm{mm})$ & TOT $(\mathrm{minu})$ \\
\hline Hyperperfusion + TND & 5 & $1.48 \pm 0.92^{\mathrm{a}}$ & $1.20 \pm 0.19^{\mathrm{a}}$ & $34.40 \pm 7.70^{\mathrm{a}}$ \\
Hyperperfusion & 11 & $1.10 \pm 0.14^{\mathrm{b}}$ & $0.93 \pm 0.15^{\mathrm{b}}$ & $32.83 \pm 3.43^{\mathrm{b}}$ \\
No hyperperfusion & 6 & $1.08 \pm 0.26^{\mathrm{c}}$ & $0.98 \pm 0.12^{\mathrm{c}}$ & $33.45 \pm 4.48^{\mathrm{c}}$ \\
\hline
\end{tabular}

D-STA: $a$ vs $b, p=0.001 ; a$ vs $c, p=0.021$; D-MCA: $a$ vs $b, p=0.028 ; a$ vs $c, p=0.012 ;$ TOT: $a$ vs $b, p=0.690 ; a$ vs $c, p=0.808$ 
Table 3. The relationship between the influence factors and the postoperative perfusion (mean $\pm S D$ ).

\begin{tabular}{lllll}
\hline Postoperative perfusion & Cases $(\mathrm{n})$ & D-STA $(\mathrm{mm})$ & D-MCA $(\mathrm{mm})$ & TOT(minu) \\
\hline Improvement & 16 & $1.22 \pm 0.24$ & $1.05 \pm 0.17$ & $33.75 \pm 5.24$ \\
No improvement & 6 & $1.08 \pm 0.26$ & $0.93 \pm 0.15$ & $32.83 \pm 3.43$ \\
P & & 0.218 & 0.252 & 0.881 \\
\hline
\end{tabular}

\section{Representative cases}

\subsection{Case 1}

A 33-year-old female presented with bilateral parietal intermittent headache for 25 years. From three years ago attacks comprising a sudden aggravating headache, dysphasia, motor deficit of bilateral upper limbs and facial sensory disturbance occurred several times per year. Each time these symptoms relieved spontaneously after 5 minutes and did not result in permanent neurologic deficits. Neurological examination found obvious memory deficits. MRI revealed only old lacuna infracts in the right occipital lobe. MRA and DSA showed severe stenosis of bilateral internal carotid artery bifurcation and moyamoya vessels on the skull base (Fig. 1). Ultrasonography examination of bilateral internal carotid arteries demonstrated a decrease in blood flow and severe stenosis (L,diameter of vessel: $2.8 \mathrm{~mm}$, PSV: $62 \mathrm{~cm} / \mathrm{s}$, EDV: $24 \mathrm{~cm} / \mathrm{s}$; R, diameter of vessel: $2.7 \mathrm{~mm}$, PSV: $64 \mathrm{~cm} / \mathrm{s}$, EDV: $26 \mathrm{~cm} / \mathrm{s}$ ). The CTP showed decreased CBF and CBV in the left frontal, temporal and parietal lobes (Fig. 2). The diagnosis was stage III moyamoya disease.

Because of severe hypoperfusion in the left hemisphere, a left STA-MCA bypass was performed after sufficient preoperative preparation. After exploration of the frontal branch and parietal branch of the left STA, the parietal branch was chosen for anastomosis and the frontal branch was electrocoagulated. The recipient artery at the M4 segment of the anterior parietal branch of the MCA was explored and, after temporoparietal craniotomy, anastomosis was performed between the stump of the STA (1.5 mm in diameter) and the M4 segment (1.0 $\mathrm{mm}$ in diameter) that supplied the parietal lobe. The patency of the anastomotic site was confirmed by ICGA and the TOT for anastomosis was 40 minutes.

The postoperative course was uneventful and systemic blood pressure was well controlled. On the second day after surgery, the patient experienced motor deficits of the right upper limb and numbness in the right face. CT scan showed no ischemic changes or hemorrhage. On the third day after the operation, CTP demonstrated that CBF compared with preoperation was increased in the left frontal and parietal area (Fig. 2). The motor deficit of the right upper limbs and numbness of the right face were completely relieved by intensive blood pressure control after 6 days and the patient was discharged without the neurologic deficit.

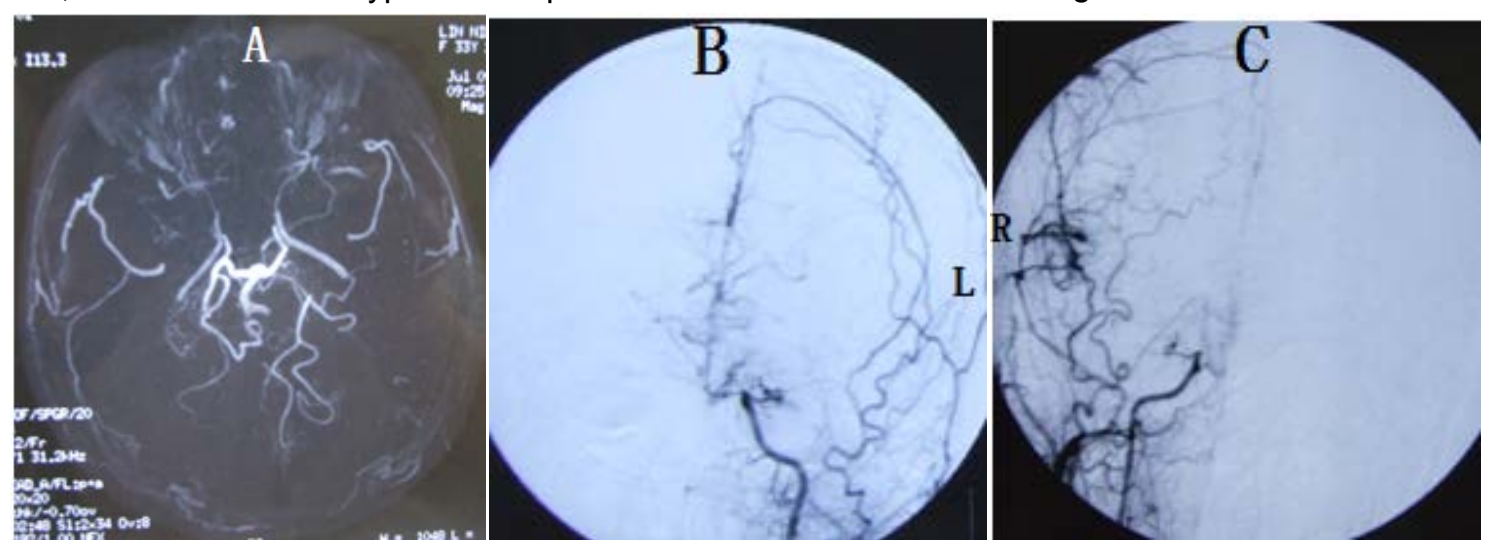


BAlet al, Possible factors influencing postoperative temporary

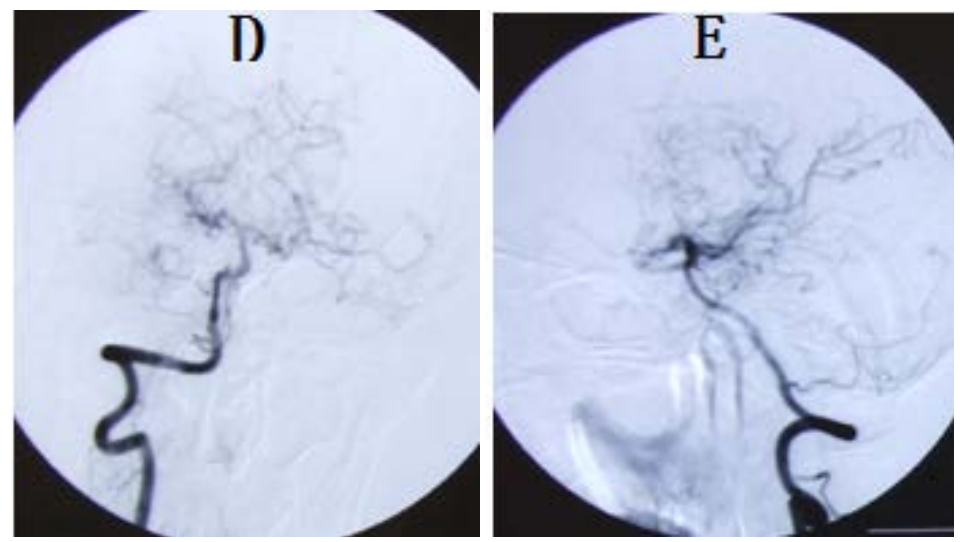

Fig.1. case 1. The severe stenosis of bilateral internal carotid artery bifurcation and moyamoya vessels on the skull base
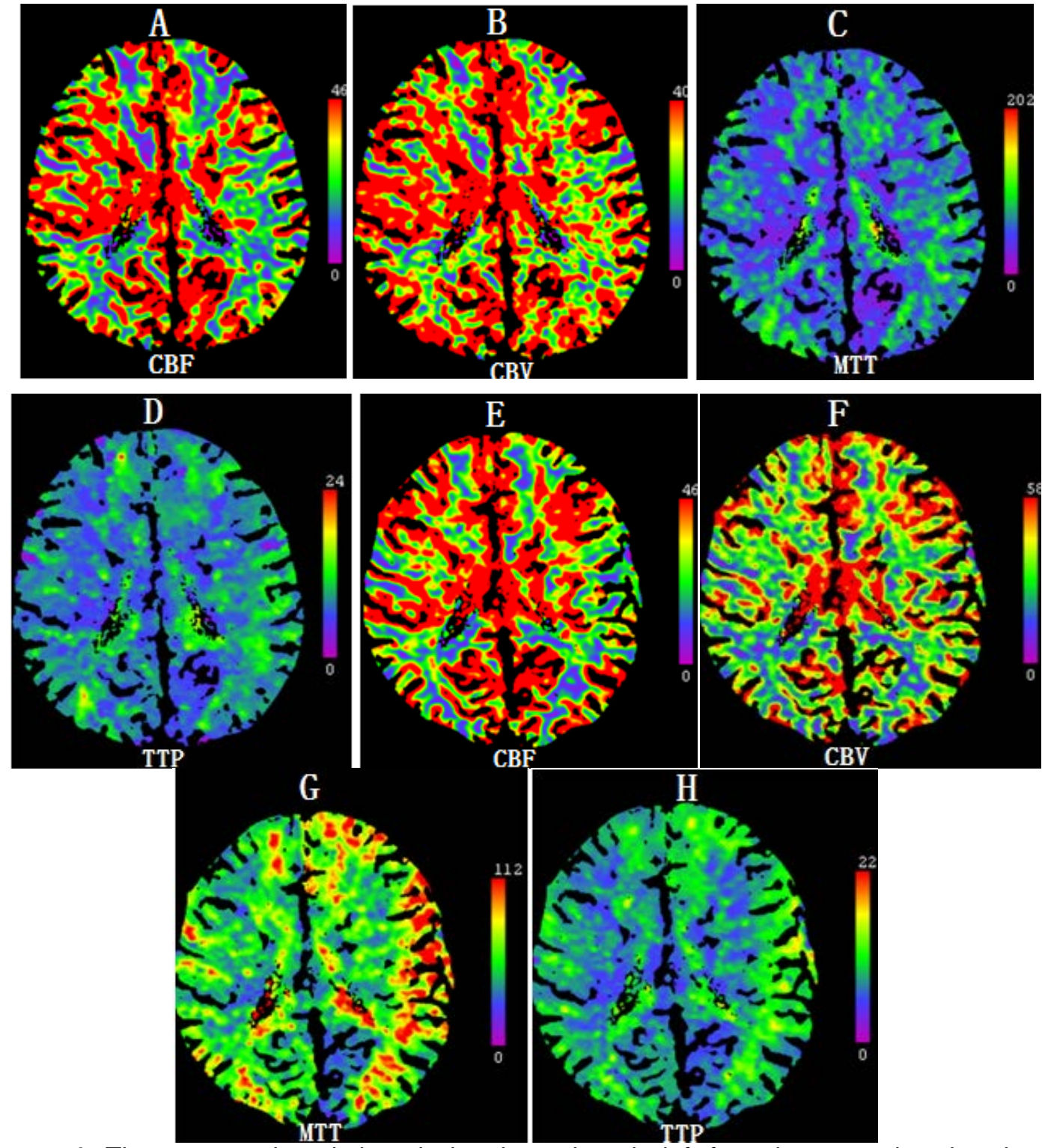

Fig.2. case1. The preoperative obvious ischemic stations in left frontal, temporal and parietal lobe were showed in CTP imaging (A, B, C and D). The regional cerebral perfusion was improved in left frontal and parietal area in CTP imaging (E, F, G and $H)$ after STA-MCA bypass. 


\subsection{Case 2}

A 43-year-male presented with 1 year of intermittent left limb numbness that generally relieved spontaneously after two to five minutes. Neurological examination found no abnormalities. MRI revealed old infarcts in bilateral frontal lobes. DSA showed a total occlusion in the branches of left internal carotid arteries and (Fig. 3). Ultrasonography examination of

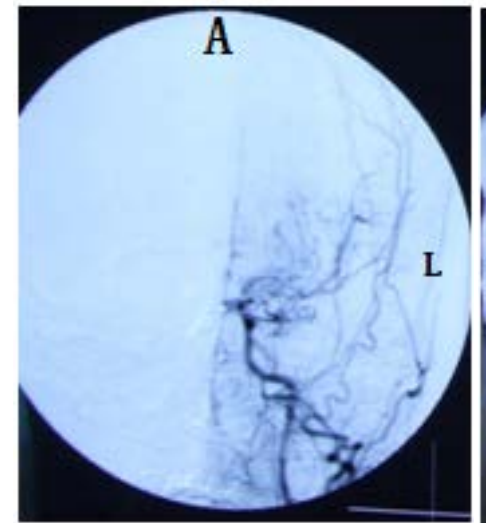

bilateral internal carotid arteries demonstrated marked decreased in blood flow in the right internal carotid artery (L, diameter of vessel: $3.5 \mathrm{~mm}$, PSV: $60 \mathrm{~cm} / \mathrm{s}$, EDV: $32 \mathrm{~cm} / \mathrm{s}$; R, diameter of vessel: $3.7 \mathrm{~mm}$, $P S V: 24 \mathrm{~cm} / \mathrm{s}$, EDV: $8 \mathrm{~cm} / \mathrm{s})$. The CTP showed that CBF and CBV were decreased in bilateral frontal and right parietal lobes (Fig. 4). The diagnosis was stage III-IV moyamoya disease.

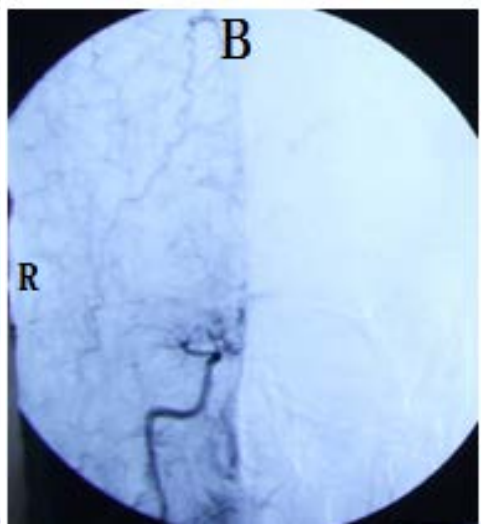

Fig.3. case2. The total occlusion in crotch of bilateral carotid artery and moyamoya vessels on skull base were showed in preoperative DSA ( $A$ and $B)$.

Right STA-MCA bypass was performed after sufficient preoperative preparation. The parietal branch was chosen for anastomosis and the frontal branch was electrocoagulated. The recipient artery at the M4 segment of the anterior frontal branch of the MCA was explored and anastomosis was performed between the stump of the STA (1.4 $\mathrm{mm}$ in diameter) and the M4 segment $(1.0 \mathrm{~mm}$ in diameter) that supplied the frontal lobe after temporoparietal craniotomy. The patency of anastomotic site was confirmed by ICGA and the TOT for anastomosis was
45 minutes.

There was no neurologic deficit on the first postoperative day. However, the patient experienced dysphasia and numbness of right face on the second day after surgery. CT scan showed no ischemic changes or hemorrhage. On the third postoperative day, CTP demonstrated increased CBF in the right frontal and parietal area (Fig. 4). The dysphasia and numbness of the right face were relieved by intensive blood pressure control after 5 days and the patient was discharged without the neurologic deficit.
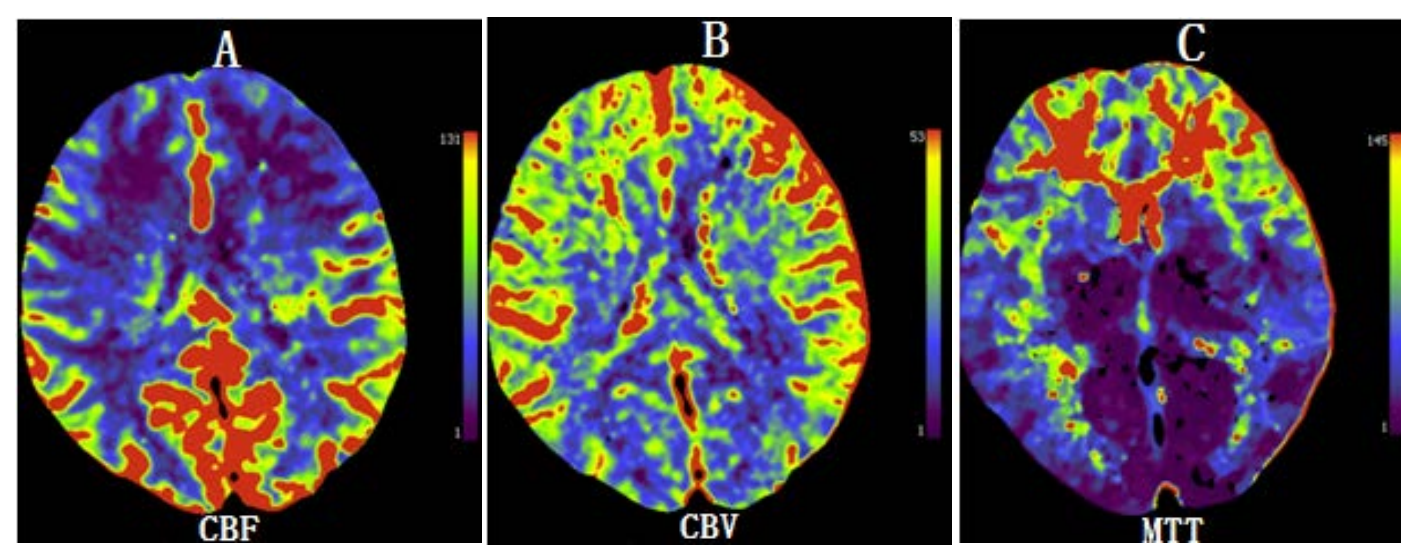


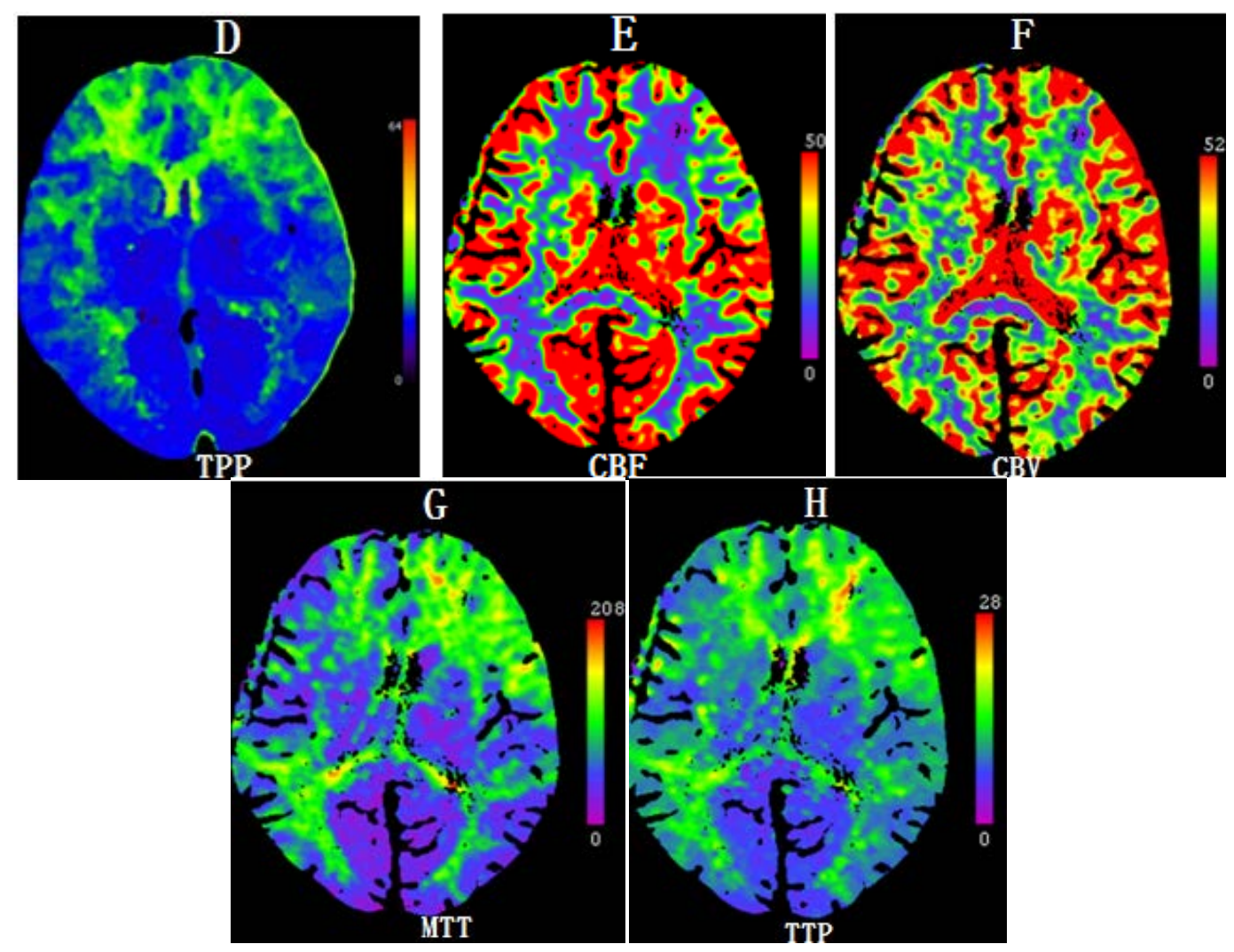

Fig.4. case2.The preoperative obvious ischemic stations in bilateral frontal and right parietal lobe were showed in CTP imaging (A, B, C and D). The regional cerebral perfusion was improved in right frontal, temporal and parietal area in CTP imaging (E, F, G and $\mathrm{H})$ after STA-MCAbypass

\subsection{Case 3}

A 54-year-female presented with asthenia of left limbs for 7 months. She had a six year history of hypertension and a 30 year history of smoking and drinking. Neurological examination found myodynamia IV, accentuation of tendon reflexes and a positive Babinski sign in the left limbs. CT scan showed multiple sheeted infarctions in the right temporal and occipital lobe (Fig. 5). DSA showed a total occlusion at the initiation site of the right internal carotid artery and stenosis of the left internal carotid artery (Fig. 5). Ultrasonography examination of bilateral internal carotid artery demonstrated no blood flow in the right internal carotid artery and the stenosis of left internal carotid artery ( $\mathrm{L}$, diameter of vessel: $3.5 \mathrm{~mm}$, PSV: $94 \mathrm{~cm} / \mathrm{s}$, EDV: $35 \mathrm{~cm} / \mathrm{s})$. The CTP showed decreased CBF and normal CBV in right frontal, temporal and parietal lobes (Fig. 6). The diagnosis was right cerebrovascular occlusive disease.

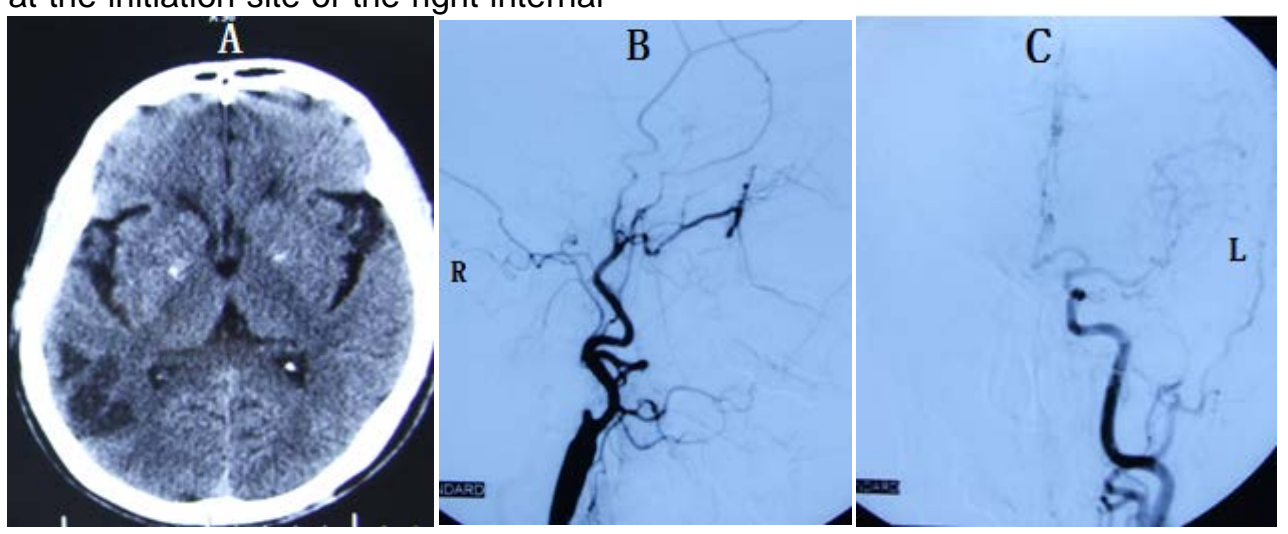

Fig.5. case3. CT scans showed multiple sheeted infractions in right temporal and occipital lobe (A). Total occlusion of initiation site of right carotid artery and the stenosis of left carotid artery were showed in DSA (B and C). 

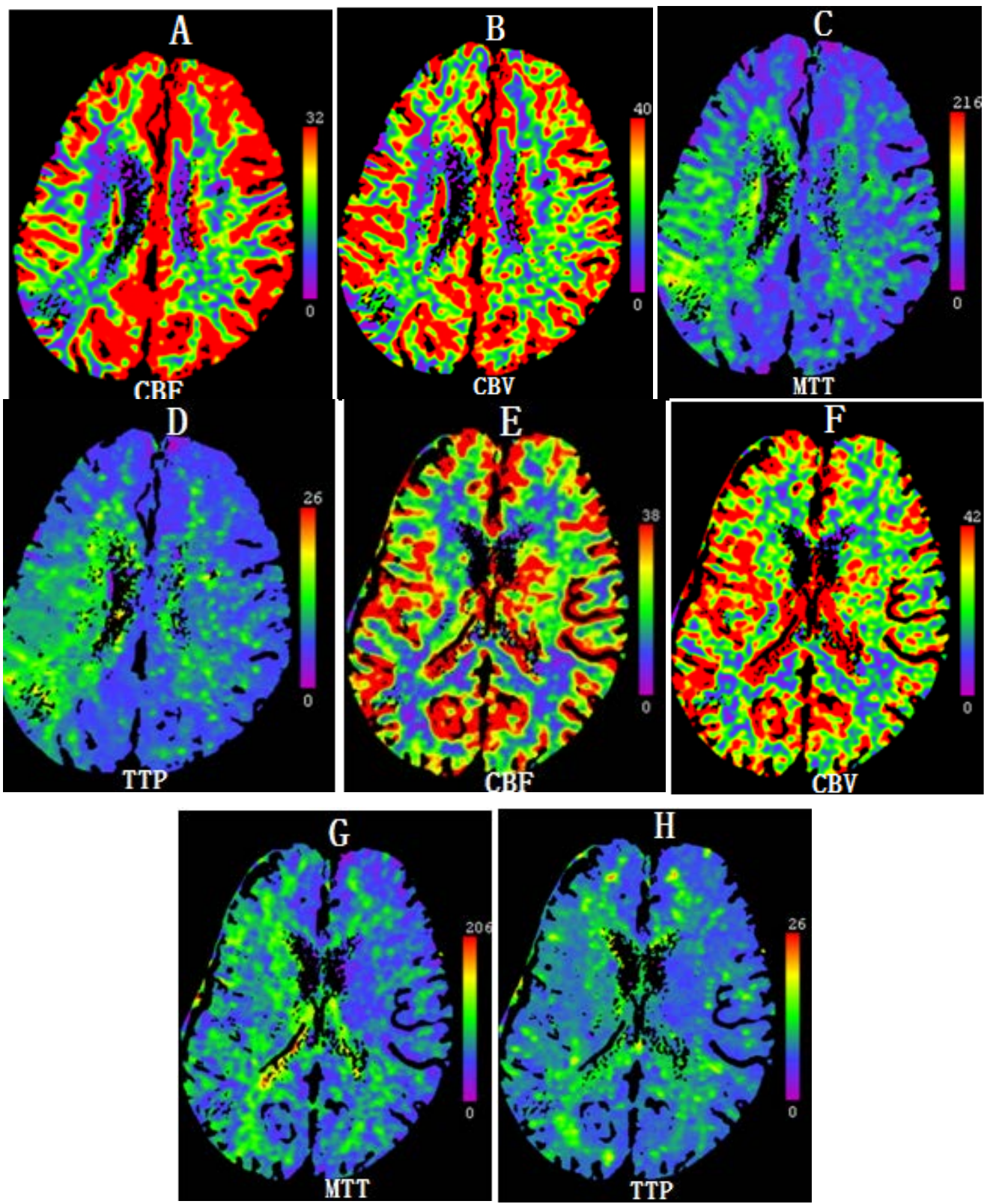

Fig.6. case3.The preoperative obvious ischemic stations in the right frontal, temporal lobe were showed in CTP imaging (A, B, C and D). The regional cerebral perfusion was improved in right frontal and temporal area in CTP imaging (E, F, G and $\mathrm{H})$ after STA-MCA bypass.

Right STA-MCA bypass was performed after preoperative preparation. The parietal branch was chosen for anastomosis and the frontal branch was electrocoagulated. The recipient artery at the M4 segment of the anterior parietal branch of the MCA was explored and anastomosis was performed between the stump of the STA (1.8 $\mathrm{mm}$ in diameter) and the M4 segment (1.4 mm in diameter) that supplied the parietal lobe. The patency of anastomotic site was confirmed by ICGA and the TOT for anastomosis was 30 minutes. experienced dysphasia, numbness and motor deficits of left upper limb. CT scan showed no fresh ischemic changes or hemorrhage. On the third postoperative day, CTP demonstrated that compared with preoperation CBF was increased in right temporal and parietal areas (Fig. 6). After seven days the dysphasia and numbness of left upper limb relieved completely, but the motor deficit of left upper limb sustained for 20 days and did not completely resolve. The neurological examination showed myodynamia IV in the left upper limb 3 months later.

On the first postoperative day, the patient 


\section{Discussion}

TND is frequently reported after the STA-MCA bypass in chronic occlusive cerebrovascular disease. Postoperative hyperperfusion is commonly regarded as the direct reason. Others have suggested that the development of TND after STA-MCA bypass may be due to the aggravation of ischemia in ischemic insults not visible in postoperative imaging. This may happen because of temporary occlusion of the recipient artery during anastomosis, sacrifice of small penetrating branches of the recipient artery, changes in blood pressure during surgery, and other effects of anesthesia. However, we could not confirm this in our patients. Our study has shown that transient improvement of regional cerebral perfusion is common $(16 / 22,72.7 \%)$ in the early postoperative period based on CTP images. In five patients TND followed the postoperative hyperperfusion and TND also correlated well with cortical dysfunction around the site of anastomosis. The TND was relieved totally after several days and coincided well with the temporal course of transient hyperperfusion. Only one patient with preoperative slight weakness of muscle in the left upper limb due to old multiple infarctions showed myodynamia IV threemonths after surgery. Fujimura et al reported that in 27 consecutive patients with adult-onset moyamoya disease who underwent STA-MCA bypass, thirteen patients (38.2\%) suffered TND for several days after surgery. They suggested that TND was due to hyperperfusion. Therefore, we suggest that postoperative transient hyperperfusion is a common phenomenon and may be one of the causes of postoperative TND after STA-MCA bypass surgery for ischemic cerebrovascular diseases.

The exact mechanism by which hyperperfusion causes TND is undetermined. During ongoing cerebral ischemia, resistant vessels dilate maximally to increase local CBF. After a long period of dilation, these vessels may become atonic and lose normal autoregulation. Excessive hemodynamic stress to the dilative vessel may trigger increased vascular permeability and induce local cerebral edema resulting in TND. Reactive oxygen species (ROS) have been also implicated in cerebral ischemia/reperfusion injury. The excessive production of ROS during revascularization may affect vascular permeability and maintenance of vascular structure.

Differences in ROS production after vascular reconstruction, vulnerability to ROS, and the expression of antioxidant enzymes in the cortex during chronic ischemia may also participate in this pathology. Ogasawara et al reported that pretreatment with the free radical scavenger edaravone prevented cerebral hyperperfusion after carotid endarterectomy in patients with atherosclerotic occlusive disease and markedly affected cerebrovascular reserve capacity. In this study, TND generally disappeared after intensive blood pressure control, indicating that changes in hemodynamic stress can be accommodated by cerebral vessels. A severe complication such as intracerebral hemorrhage is rare in STA-MCA bypass. The STA-MCA bypass is a safe and effective method for ischemic cerebral vascular disease.

In our study, the CTP was used to determine the regional cerebral perfusion and the change in CTP was evaluated by comparison with not non-operated side. The CTP is a relatively new technique that allows rapid qualitative and quantitative evaluation of cerebral perfusion. Although the accuracy of quantitative results and the reproducibility of perfusion CT have not been fully validated, CTP has been found to be useful for noninvasive diagnosis of cerebral ischemia and infarction and was introduced as a means to rapidly and easily evaluate cerebral perfusion in patients presenting with acute stroke symptoms. Recently, intraoperative quantitative measurements of bypass flow using an ultrasonic flow probe have been reported. Transcranial Doppler ultrasound (TCD) is a common method that uses an ultrasonic flow probe to measure the bypass flow which allows non-invasive, beat-by-beat measurement of changes in the vessel's blood velocity. However, the major drawback of TCD is that it can only be used during the operation due to the effect of cranial bone. Vascular spasms were not considered in measuring CBF during the operation. Laser Doppler flowmetry (LDF) is an established technique for the real-time measurement of microvascular red blood cell perfusion in tissue. Kawamata et al reported that intraoperative rCBF measurement using laser Doppler flowmetry may predict a risk of post-EC-IC bypass cerebral hyperperfusion in moyamoya disease. The limitations of laser doppler flowmetry were that the outcomes were influenced commonly by large vessels near the ultrasonic probe. In addition, displacement of the ultrasonic probe can cause an inaccurate measurement of CBF. Cerebral perfusion can also be measured by various tools including PET, magnetic resonance imaging, single photon emission computed tomography and xenon CT.

Not all patients with regional cerebral perfusion develop TND; in our study the incidence was $31.3 \%$ (5/16). The anatomical vascular structures around the site of the anastomosis may play an important role and should be considered carefully. We used ICGA to provide a reliable and rapid intraoperative assessment of bypass patency. It has a high spatial resolution and facilitates the identification of stenoses and obstruction at the anastomotic site or vascular obstructions. The key factors determining blood flow through the site of the anastomosis are the supplied -19 - 
artery and recipient artery used for the revascularization. Recent evidence suggests that STA-MCA anastomosis for moyamoya disease, which usually provides low-flow revascularization because of the relatively small diameter of the recipient artery, could also result in TND. We investigated the diameter of the STA and MCA (M4) in 22 patients with STA-MCA bypass (Table 1) and found that the diameter of the STA and MCA (M4) in the patients with TND was significantly larger than in the patients without TND (Table 2). We speculated that the diameter of the STA and MCA may be major factors influencing the development of TND in STA-MCA bypass. In contrast to carotid endarterectomy and extracranial-to-intracranial internal carotid artery bypass surgery, STA-MCA anastomosis rarely causes permanent neurologic deficits, probably because the bypass flow through the STA is rather less than the flow through the ICA. In patients with larger superficial temporal and middle cerebral arteries it is easy to induce local cerebral edema resulting in TND because of the increased blood flow through the site of the anastomosis. The goal of revascularization is to supply an adequate blood flow to improve the perfusion. Although a larger diameter of the supplied and recipient arteries is necessary for the success of surgery, it increases the risk of TND. However, use of the larger blood vessel in STA-MCA bypass may be a reason for the high incidence of TND (22.7\%). Measuring the diameter of the STA and MCA would be of great value to clarify the predictive factors for postoperative TND and to conduct its adequate management such as intensive blood pressure control. The TOT for recipient artery was investigated and was not obviously related to TND. The limited number of cases in the present study did not allow us to completely determine the factors underlying TND after STA-MCA bypass surgery. We will accumulate more cases to elucidate this issue in future studies.

In conclusion, postoperative transient hyperperfusion is a common phenomenon and may be one of the causes of postoperative temporary neurological deterioration after STA-MCA bypass surgery for ischemic cerebrovascular diseases. The diameter of STA and diameter of MCA may be the factors influencing on the temporary neurologic deterioration frequently observed after STA-MCA bypass.

\section{Reference}

Jeffree RL, Stoodley MA. STA-MCA bypass for symptomatic carotid occlusion and haemodynamic impairment. J Clin Neurosci 2009;16:226-35.

is associated with preoperative hemodynamic impairment and intraoperative cerebral ischemia. J Cereb Blood Flow Metab 2006;26:878-84.

Ogasawara K, Yukawa H, Kobayashi M, et al. Prediction
Tsai ST, Yen PS, Wang YJ, et al. Superficial temporal artery-middle cerebral artery bypass for ischemic atherosclerotic middle cerebral artery disease. J Clin Neurosci 2009;16:1013-7.

Hwang G, Oh CW, Bang JS, et al. Superficial temporal artery to middle cerebral artery bypass in acute ischemic stroke and stroke in progress. Neurosurgery 2011;68:723-9.

Amin-Hanjani S, Butler WE, Ogilvy CS, et al. Extracranialintracranial bypass in the treatment of occlusive cerebrovascular disease and intracranial aneurysms in the United States between 1992 and 2001: a population-based study. J Neurosurg 2005;103:794804.

Vilela MD, Newell DW. Superficial temporal artery to middle cerebral artery bypass: past, present, and future. Neurosurg Focus 2008; 24:E2: 1-9.

Houkin K, Ishikawa T, Yoshimoto T, et al. Direct and indirect revascularization for moyamoya disease surgical techniques and peri-operative complications. Clin Neurol Neurosurg 1997;99 Suppl 2:S142-S145.

Fujimura M, Kaneta T, Mugikura S, et al. Temporary neurologic deterioration due to cerebral hyperperfusion after superficial temporal artery-middle cerebral artery anastomosis in patients with adult-onset moyamoya disease. Surg Neurol 2007;67:273-82.

Chater N, Popp J: Microsurgical vascular bypass for occlusive cerebrovascular disease; review of 100 cases. Surg Neurol 1976; 6:115-8.

Heros RC, Scott RM, Kistler JP, et al. Temporary neurological deterioration after extracranial-intracranial bypass. Neurosurgery 1984;15:178-185.

Furuya K, Kawahara N, Morita A, et al. Focal hyperperfusion after superficial temporal artery-middle cerebral artery anastomosis in a patient with moyamoya disease. Case report. J Neurosurg 2004;100:128-32.

Kim JE, Oh CW, Kwon OK, et al. Transient hyperperfusion after superficial temporal artery/middle cerebral artery bypass surgery as a possible cause of postoperative transient neurological deterioration. Cerebrovasc Dis 2008;25:580-6.

Fujimura M, Mugikura S, Kaneta T, et al. Incidence and risk factors for symptomatic cerebral hyperperfusion after superficial temporal artery-middle cerebral artery anastomosis in patients with moyamoya disease. Surg Neurol 2009;71:442-447.

Fujimura M, Tominaga T, Chan PH: Neuroprotective effect of an antioxidant in ischemic brain injury: involvement of neuronal apoptosis. Neurocrit Care 2005;2:59-66.

Komoribayashi N, Ogasawara K, Kobayashi M, et al. Cerebral hyperperfusion after carotid endarterectomy

and monitoring of cerebral hyperperfusion after carotid endarterectomy by using single-photon emission computerized tomography scanning. J Neurosurg 2003;99:504-10. 
Yoshimoto T, Houkin K, Kuroda S, et al. Low cerebral blood flow and perfusion reserve induce hyperperfusion after surgical revascularization: case reports and analysis of cerebral hemodynamics. Surg Neurol 1997;48:132-8.

Horn P, Scharf J, Peña-Tapia P, et al. Risk of intraoperative ischemia due to temporary vessel occlusion during standard extracranial-intracranial arterial bypass surgery. J Neurosurg 2008;108:464-9.

Higashi $\mathrm{S}$, Matsuda $\mathrm{H}$, Fujii $\mathrm{H}$, et al. Luxury perfusion syndrome confirmed by sequential studies of regional cerebral blood flow and volume after extracranial to intracranial bypass surgery: case report. Neurosurgery 1989;25:85-9.

Chan PH: Role of oxidants in ischemic brain damage. Stroke 1996;27:1124-9.

Fujimura M, Shimizu H, Mugikura S, et al. Delayed intracerebral hemorrhage after superficial temporal artery-middle cerebral artery anastomosis in a patient with moyamoya disease: possible involvement of cerebral hyperperfusion and increased vascular permeability. Surg Neurol 2009;71:223-7.

Ogasawara K, Inoue T, Kobayashi M, et al. Pretreatment with the free radical scavenger edaravone prevents cerebral hyperperfusion after carotid endarterectomy. Neurosurgery 2004;55:1060-7.
Wang JT, Zhang D, Zuo F, et al. Application of indocyanine green angiography in bypass surgery for moyamoya disease. Zhonghua Yi Xue Za Zhi 2010;90:1628-30.

Awano T, Sakatani K, Yokose N,et al. Intraoperative EC-IC bypass blood flow assessment with indocyanine green angiography in moyamoya and non-moyamoya ischemic stroke. World Neurosurg;73:668-74.

Woitzik J, Horn P, Vajkoczy P, et al. Intraoperative control of extracranial-intracranial bypass patency by nearinfrared indocyanine green videoangiography. J Neurosurg 2005;102:692-8.

Baron JC, Bousser MG, Rey A, et al. Reversal of focal "misery-perfusion syndrome" by extra-intracranial arterial bypass in hemodynamic cerebral ischemia. A case study with 150 positron emission tomography. Stroke 1981;12:454-9.

Ogasawara K, Komoribayashi N, Kobayashi M, et al. Neural damage caused by cerebral hyperperfusion after arterial bypass surgery in a patient with moyamoya disease: case report. Neurosurgery 2005;56:E1380.

Morgan MK, Ferch RD, Little NS, et al. Bypass to the intracranial internal carotid artery. J Clin Neurosci 2002;9:418-24. 